\title{
Dementia Worries and Cognitive Complaints in a Long-Life Society
}

\author{
Eva-Marie Kessler
}

Department of Psychology, MSB Medical School Berlin, Germany

Through many channels of communication - television, radio, the internet, one's own personal social environmentpeople of all ages are today increasingly being confronted with the subject of dementia. The diagnosis of dementia in celebrities such as Margaret Thatcher, Malcolm Young, or Robin Williams received considerable media attention. Dementia is also increasingly being featured in documentaries or feature films, examples being "Still Alice," "Away from Her," or "The Savages." In addition, science journalism frequently provides novel insights on prevention and treatment options, thus putting the topic of dementia even more in the focus of media. Contributing to the growing media presence of dementia is also the fact that people with dementia themselves have started to go public more frequently. Richard Taylor, a Texan professor of psychology who lived well with dementia for more than a decade before dying in 2015, achieved particular prominence. Furthermore, parallel to increasing life expectancy, the absolute number of persons afflicted with dementia has been increasing worldwide, resulting in more and more people being directly or indirectly socially affiliated with people affected by the disease. Also rising is the number of people who either professionally or voluntarily support and care for people with dementia.

As much as dementia is becoming an increasingly salient event in the daily life of individuals in our "long-life society," the likelihood of individuals experiencing "memory lapses" and other problems of cognitive functioning - often called subjective cognitive impairment (SCI; Rabin et al., 2015) - may also increase, because of

1) objective cognitive decline during the development of dementia;

2) nonorganic mental illness, particularly major depression;

3) experienced worries or even anxiety regarding a possible future dementia diagnosis and associated high levels of psychological stress and self-awareness.

Such fears and worries regarding dementia - often called dementia worry (DW; Kessler et al., 2012) - tend to occur more frequently in people who experience objective cognitive problems. Yet, there are individuals of all ages who are afraid of developing dementia, despite the lack of any subjective or objective cognitive problems.

Despite the significant overlap between SCI and DW, the two scientific constructs have largely been researched separately and by different scientific communities, though the empirical research spans less than two decades in both cases. Specifically, research on SCI stands in the tradition of epidemiological and gerontopsychiatric research, focusing mainly on investigating SCI as a possible predictor of future dementia, both in community-based and in clinical settings (e.g., Jessen, 2014). Research on DW, on the other hand, is more strongly associated with the tradition of social gerontology and geropsychology (e.g., Cutler \& Hodgson, 2001; Werner, 2002). The populations investigated were firstdegree relatives of people with dementia, medical or nursing staff, and "the worried well," that is, people who seek rigorous dementia screening and other dementia-related help in the absence of dementia symptoms in memory clinics, etc.

In the case of SCI, the research impetus was primarily focused on identifying early indicators of dementia in the service of prevention, while in the case of DW the focus lay on informing individuals about dementia and supporting them in dealing with associated fears and stigmatization. A construct referred to in both lines of research is that of depression. And indeed, there is evidence for a positive association between depressive symptoms and SCI and DW, respectively.

This special issue brings together the two research traditions, with the goal of improving future research on possible conceptional and empirical associations between SCI and DW. Readers should be aware that there is no consistent terminology regarding SCI or DW; this is also reflected in the six contributions. Other commonly used terms for SCI are subjective cognitive complaints, subjective memory impairment, and anticipatory dementia. Dementia-related anxiety and fear of Alzheimer's disease are different expressions for DW. 
Maxfield, Cui, et al. (2020) hypothesized that increased awareness regarding Alzheimer's disease and related dementias (ADRD) could further dementia-related anxiety. They examine variables that may predict a person's interest in ADRD testing. Their findings suggest that variables such as a subjectively perceived high risk of developing ADRD, a family history of dementias, and existing dementia-related anxiety predicted a greater interest in being tested for $\mathrm{ADRD}$, suggesting that higher awareness and higher levels of dementia-related anxiety are associated.

In addition to increased awareness, stigma and negative perceptions of dementias may also further subjective concerns regarding the development of ADRD. Maxfield and Greenberg (2020) expected higher levels of ADRD stigma to be associated with higher anxiety regarding the development of dementia. Middle-aged and older subjects completed an online survey, and the results hinted that ADRD exposure, self-perceived risk, and anticipated stigma are significantly associated with dementia-related anxiety.

Over time, the construct of dementia worry seems moderately stable, as shown by Martin et al. (2020). Conceptualizing a 2-year longitudinal online study, the authors examined both the perception of personal dementia risk and DW, finding changes in DW unrelated to perceived risk or family history. In contrast, perceived dementia risk was positively associated with preceding DW. However, the complexity of both DW and the perception of personal dementia risk requires further research to differentiate and fully understand influencing factors.

Maxfield, Roberts, et al. (2020) did just that by focusing on two clients who reported anxiety about their cognitive functionality while seeking out a neuropsychological assessment, thus contributing to a better understanding of aspects furthering dementia-related anxiety. While both clients reported negative emotions regarding forgetfulness and social repercussions, only one of them met the criteria for a mild neurocognitive disorder. That client voiced concerns about the declining quality of life with Alzheimer's disease. For the second case, the anxiety regarding cognitive status could most likely be attributed to a generalized anxiety disorder. Those findings underline that dementiarelated anxiety may stem from various roots, have individual social consequences, and thus demand individualized interventions.

Depression and depressive symptoms have been of interest in both DW- and SCI-related research. Kessler et al. (2020) investigated depressive symptoms in memory-clinic patients. While younger age, lower levels of education, and low sleep quality were found to be predictors of depressive symptoms, subjective cognitive complaints (SCC) remained insignificant in predicting depressive symptoms. Therefore, memory clinics should also be regarded in their gatekeeping function to better manage depression.
The impact a major depression may have on the extent and quality of experienced SCI was investigated by Soellner et al. (2020). Their sample consisted of patients with and without symptoms of a major depressive disorder who sought consultation at a memory clinic. Negative affect and SCI levels were significantly higher in patients suffering from major depression compared to those without. In patients without major depression, the psychoaffective state significantly predicted their SCI levels. Since those who suffer from major depression are at a higher risk of suffering from cognitive impairment than patients who do not, the affective state should be accounted for when assessing SCI. Those findings also underline that intersectional research on the interactions between depressive symptoms and cognitive functionality can further a better understanding of both constructs.

We hope that readers enjoy the diverse perspectives on DW and SCI presented by our authors, and that they may serve as a catalyst for expanding basic and applied research on DW and SCI in new and synergistic directions.

\section{References}

Cutler, S. J., \& Hodgson, L. G. (2001). Correlates of personal concerns about developing Alzheimer's disease among middleaged persons. American Journal of Alzheimer's Disease and Other Dementias, 16(6), 335-343. https://doi.org/10.1177/ 153331750101600604

Jessen, F. (2014). Subjective and objective cognitive decline at the pre-dementia stage of Alzheimer's disease. European Archives of Psychiatry and Clinical Neuroscience, 264(S1), 3-7. https:// doi.org/10.1007/s00406-014-0539-z

Kessler, E.-M., Bowen, C. E., Baer, M., Froelich, L., \& Wahl, H.-W. (2012). Dementia worry: A psychological examination of an unexplored phenomenon. European Journal of Ageing, 9, 275284. https://doi.org/10.1007/s10433-012-0242-8

Kessler, E., Frank, V., Klostermann, A., Peters, O., \& Schäfer, T. (2020). Concomitants of depressive symptoms in memory clinic patients. GeroPsych, 34(1), 37-44. https://doi.org/10.1024/ 1662-9647/a000231

Martin, K., Lang, F., Rupprecht, R., \& Nömer, J. (2020). Dementia worry and the perception of personal risk. GeroPsych, 34(1), 23-30. https://doi.org/10.1024/1662-9647/a000247

Maxfield, M., Cui, R., Roberts, J., \& Fiske, A. (2020). Interest in dementia testing. GeroPsych, 34(1), 5-11. https://doi.org/ 10.1024/1662-9647/a000230

Maxfield, M., \& Greenberg, J. (2020). Anticipated stigma and dementia-related anxiety in middle-aged and older adults. GeroPsych, 34(1), 13-22. https://doi.org/10.1024/1662-9647/ a000234

Maxfield, M., Roberts, J., \& Dieker, J. (2020). Case Studies in Dementia-Related Anxiety. GeroPsych, 34(1), 31-36. https:// doi.org/10.1024/1662-9647/a000243

Rabin, L. A., Smart, C. M., Crane, P. K., Amariglio, R. E., Berman, L. M., Boada, M., Buckley, R. F., Chételat, G., Dubois, B., Ellis, K. A., Gifford, K. A., Jefferson, A. L., Jessen, F., Katz, M. J., Lipton, R. B., Luck, T., Maruff, P., Mielke, M. M., Molinuevo, J. L., ... Sikkes, S. A. (2015). Subjective cognitive decline in older adults: An overview of self-report measures used across 
19 international research studies. Journal of Alzheimer's Disease: JAD, 48(Suppl 1), S63-S86. https://doi.org/10.3233/ JAD-150154

Soellner, A., Lubitz, A., Lueschow, A., \& Niedeggen, M. (2020). Predictors of subjective cognitive impairment in help-seekers with and without symptoms of a major depression. GeroPsych, 34(1), 45-53. https://doi.org/10.1024/1662-9647/a000232

Werner, P. (2002). Assessing correlates of concern about developing Alzheimer's dementia among adults with no family history of the disease. American Journal of Alzheimer's Disease and Other Dementias, 17(6), 331-337. https://doi.org/10.1177/ 153331750201700609

\section{Prof. Dr. habil. Eva-Marie Kessler}

Rüdesheimer Str. 50

14197 Berlin

Germany

eva-marie.kessler@medicalschool-berlin.de 